\title{
COMPARISON THEOREMS ON THE OSCILLATION AND ASYMPTOTIC BEHAVIOUR OF HIGHER-ORDER NEUTRAL DIFFERENTIAL EQUATIONS
}

\author{
BAŞAK KARPUZ, ÖZKAN ÖCALAN and SERMIN ÖZTÜRK \\ Department of Mathematics, Faculty of Science and Arts, ANS Campus, Afyon Kocatepe University, \\ 03200 Afyonkarahisar, Turkey \\ e-mail:bkarpuz@gmail.com,ozkan@aku.edu.tr,ssahin@aku.edu.tr \\ URLs: http://www2.aku.edu.tr/ bkarpuz (B. Karpuz), \\ http://www2.aku.edu.tr/ ozkan (Ö. Öcalan)
}

(Received 20 November 2008; accepted 29 May 2009)

\begin{abstract}
In this work, oscillatory and asymptotic behaviours of all solutions of higher-order neutral differential equations are compared with first-order delay differential equations, depending on two different ranges of the coefficient associated with the neutral part. Some simple examples are given to compare our results with the existing results in the literature and to illustrate the significance and applicability of our new results. Our results generalise, improve and correct some of the existing results in the literature.
\end{abstract}

2000 Mathematics Subject Classification. 34K11, 34C15.

1. Introduction. In the natural sciences, technology and population dynamics, neutral delay differential equations find many application fields. For instance neutral delay differential equations appear in modelling of the networks containing lossless transmission lines (as in high-speed computers in which the lossless transmission lines are used to interconnect switching circuits), in the study of vibrating masses attached to an elastic bar, as Euler-type equations in some variational problems, in the theory of automatic control and in neuro-mechanical systems in which inertia plays a major role (see $[\mathbf{1 0}])$. The readers are referred to $[1,7,9,10,12]$ for fundamental results on the oscillation theory of differential equations.

In this paper, we consider the higher-order neutral differential equations of the following type:

$$
[x(t)+p(t) x(\tau(t))]^{(n)}+q(t) x(\sigma(t))=0 \quad \text { for } t \in\left[t_{0}, \infty\right),
$$

where $t_{0} \in \mathbb{R}, p \in C\left(\left[t_{0}, \infty\right), \mathbb{R}\right), q \in C\left(\left[t_{0}, \infty\right), \mathbb{R}^{+}\right)$and $\tau, \sigma \in C\left(\left[t_{0}, \infty\right), \mathbb{R}\right)$ satisfy $\lim _{t \rightarrow \infty} \tau(t)=\lim _{t \rightarrow \infty} \sigma(t)=\infty$ and $\tau(t), \sigma(t) \leq t$ for all $t \in\left[t_{0}, \infty\right)$. In the sequel, we consider the following two ranges of the coefficient $p$ :

(R1) $p \in C\left(\left[t_{0}, \infty\right), \mathbb{R}^{+}\right)$satisfies $\bar{\ell}_{p}:=\limsup _{t \rightarrow \infty} p(t)<1$,

(R2) $p \in C\left(\left[t_{0}, \infty\right), \mathbb{R}^{-}\right)$satisfies $\underline{\ell}_{p}:=\liminf _{t \rightarrow \infty} p(t)>-1$.

In a very recent paper [28], the authors applied a new method to obtain the following result for (1) with even order. 
TheOREM A [28, Theorem 1]. Let $p \in C\left(\left[t_{0}, \infty\right), \mathbb{R}^{+}\right)$satisfy $0 \leq p(t) \leq 1$ for all sufficiently large $t$. If there exists a constant $\lambda_{0} \in(0,1)$ such that every solution of the first-order delay differential equation

$$
y^{\prime}(t)+\frac{\lambda_{0}}{(n-1) !}(\sigma(t))^{n-1}[1-p(\sigma(t))] q(t) y(\sigma(t))=0 \quad \text { for } t \in\left[t_{0}, \infty\right)
$$

is oscillatory, then every solution of (1) with even order is also oscillatory.

The proof of the theorem stated above is very nice and simple for even-order neutral differential equations; however, for odd-order and/or higher-order neutral differential equations with the ranges (R1) and/or (R1), the proof is not straightforward. For odd-order delay differential equations, the readers may find very interesting results in the papers $[\mathbf{2}, \mathbf{4}, \mathbf{5}, \mathbf{8}, \mathbf{2 0}, \mathbf{2 7}]$, and we wish to point out here that all these mentioned papers study (1) with (R2). To the best of our knowledge, [6, 15-19] are the only papers including some results for (1) for arbitrary-order neutral delay differential equations. Since the conditions in the papers mentioned above are different from ours, our technique is completely different, and it is indeed interesting to point out that our results are not directly comparable with their results. However, we provide two simple examples such that almost all of the results of these mentioned papers fail to apply; we therefore feel that our results are a little bit weaker in a sense in some cases.

In the sequel, we need to state a modification of the most suitable results in [6, 15-19] for (1) to compare our results.

Theorem B (see [15, Theorem 3.5] and [19, Theorem 2]). Assume that $p$ satisfies (R1) or (R2). Then every bounded solution of (1) oscillates or tends to zero asymptotically if $\int^{\infty} s^{n-1} q(s) \mathrm{d} s=\infty$ holds. However, the conclusion holds only with oscillation when $n$ is even and p satisfies (Rl).

TheOREM C (see [15, Theorem 3.6]). Assume that p holds one of the conditions (R1) or (R2). Then every solution of (1) oscillates or tends to zero asymptotically if $\int^{\infty} s^{n-2} q(s) \mathrm{d} s=\infty$ holds. This conclusion holds only with oscillation when $n$ is even and p satisfies $(R I)$.

Set $t_{-1}:=\inf _{t \in\left[t_{0}, \infty\right)}\{\tau(t), \sigma(t)\}$. A function $x$ is called to be solution of (1) provided that $x \in C\left(\left[t_{-1}, \infty\right), \mathbb{R}\right), x+p(t) x \circ \tau \in C^{n}\left(\left[t_{0}, \infty\right), \mathbb{R}\right)$, and $x$ satisfies (1) identically on $\left[t_{0}, \infty\right)$. In the sequel, for convenience, we only restrict our attention to solutions of (1), which does not vanish on any sub-half-line of $\left[t_{0}, \infty\right)$. As is customary, a solution $x$ of (1) is said to be oscillatory if it has arbitrarily large zeros; otherwise, it is called non-oscillatory; and (1) is called oscillatory if all its solutions are oscillatory and almost oscillatory if all its solutions are oscillatory or convergent to zero asymptotically.

This paper is arranged in the following form: in Section 2, we give some useful lemmas; in Section 3, we state our main results on (1) with $p$ satisfying the condition (R1) and/or the condition (R2); in Section 4, we give some applications; finally, in Section 5, we make our final comments to finalise the paper.

2. Auxiliary lemmas. The Kneser's theorem is stated below, the readers may find this result in [1, Lemma 2.2.1] and [12, Lemma 5.2.1], which is one of the most useful tools in the oscillation of higher-order delay equations.

LEMma 1 (Kneser's theorem). Let $f \in C^{n}\left(\left[t_{0}, \infty\right), \mathbb{R}\right)$ be a function of fixed sign such that $f^{(n)}$ is of fixed sign and not identically zero on a subray of $\left[t_{0}, \infty\right)$. Then, there 
exist $m \in \mathbb{Z}$ and $t_{1} \in\left[t_{0}, \infty\right)$ such that $0 \leq m \leq n-1$, and $(-1)^{n+m} f f^{(n)} \geq 0$,

$$
f f^{(j)}>0 \text { for } j=0,1, \ldots, m-1 \text { when } m \geq 1
$$

and

$$
(-1)^{m+j} f f^{(j)}>0 \quad \text { for } j=m, m+1, \ldots, n-1 \text { when } m \leq n-1
$$

hold on $\left[t_{1}, \infty\right)$.

The following lemma is taken from [1, Lemma 2.2.3].

LemMA 2. Let $f$ be a function as in Kneser's theorem. If $\lim _{t \rightarrow \infty} f(t) \neq 0$, then for every $\lambda \in(0,1)$, there exists $t_{2} \in\left[t_{1}, \infty\right)$ such that

$$
|f| \geq \frac{\lambda}{(n-1) !} t^{n-1}\left|f^{(n-1)}\right|
$$

holds on $\left[t_{2}, \infty\right)$. Here, $t_{2}=t_{2}(\lambda) \rightarrow \infty$ as $\lambda \rightarrow 1$.

Next, we prove the following useful lemma, which helps us in our proofs.

LEMma 3. Let $f$ and $g \in C\left(\left[t_{0}, \infty\right), \mathbb{R}\right)$ and $\alpha \in C\left(\left[t_{0}, \infty\right), \mathbb{R}\right)$ satisfies $\lim _{t \rightarrow \infty} \alpha(t)=\infty$ and $\alpha(t) \leq t$ for all $t \in\left[t_{0}, \infty\right)$; further suppose that there exists $h \in C\left(\left[t_{-1}, \infty\right), \mathbb{R}^{+}\right)$, where $t_{-1}:=\min _{t \in\left[t_{0}, \infty\right)}\{\alpha(t)\}$, such that $f(t)=h(t)+$ $g(t) h(\alpha(t))$ holds for all $t \in\left[t_{0}, \infty\right)$. Suppose that $\ell_{f}:=\lim _{t \rightarrow \infty} f(t)$ exists and $\underline{\ell}_{g}:=\liminf \operatorname{in}_{t \rightarrow \infty} g(t)>-1$. Then $\bar{\ell}_{h}:=\lim \sup _{t \rightarrow \infty} h(t)>0$ implies $\ell_{f}>0$.

Proof. Let $t_{1} \in\left[t_{0}, \infty\right)$ and $0 \geq m_{g}>-1$ satisfy $g(t) \geq m_{g}$ for all $t \in\left[t_{1}, \infty\right)$; then we may pick an increasing divergent $\left\{\xi_{k}\right\}_{k \in \mathbb{N}} \subset\left[t_{1}, \infty\right)$ such that $\lim _{k \rightarrow \infty} h\left(\xi_{k}\right)=\bar{\ell}_{h}$ holds. First, we shall consider the case that $h$ is unbounded; then we may suppose that $x\left(\xi_{k}\right)=\max \left\{x(t): t \in\left[t_{1}, \xi_{k}\right]\right\}$ holds for all $k \in \mathbb{N}$. Then, we have $f\left(\xi_{k}\right) \geq h\left(\xi_{k}\right)+$ $m_{g} h\left(\alpha\left(\xi_{k}\right)\right) \geq\left(1+m_{g}\right) h\left(\xi_{k}\right)$ for all $k \in \mathbb{N}$, which implies $\ell_{f}=\infty$ by letting $k$ tend to infinity. Next, let $h$ be bounded; then we may suppose that $\lim _{k \rightarrow \infty} h\left(\alpha\left(\xi_{k}\right)\right)=L_{h}$ for some $L_{h} \geq 0$. Note that $L_{h} \leq \bar{\ell}_{h}$ is true. Therefore, following similar arguments as that in the previous case, we have $\ell_{f} \geq \bar{\ell}_{h}+m_{g} L_{h} \geq \bar{\ell}_{h}\left(1+m_{g}\right)>0$. The proof is hence completed.

\section{Main results.}

THEOREM 1. Assume that $p$ satisfies the condition (R1), and (2) is oscillatory for some $\lambda_{0} \in(0,1)$. Then (1) is almost oscillatory.

Proof. Let $x$ be a non-oscillatory solution of (1), which does not tend to zero asymptotically. Clearly, $x$ can be assumed to be eventually positive without loss of generality, since (1) is linear. Say $x(t), x(\tau(t)), x(\sigma(t))>0$ for all $t \in\left[t_{1}, \infty\right)$ for some sufficiently large $t_{1} \in\left[t_{0}, \infty\right)$. Set

$$
y_{x}(t):=x(t)+p(t) x(\tau(t)) \text { for } t \in\left[t_{1}, \infty\right) .
$$

Then, we see that

$$
y_{x}^{(n)}(t)=-q(t) x(\sigma(t)) \leq 0 \text { for all } t \in\left[t_{1}, \infty\right)
$$


which indicates that $y_{x}^{(j)}$ is strictly monotonic and of single sign on $\left[t_{2}, \infty\right)$ for $j=$ $0,1, \ldots, n-1$, where $t_{2} \in\left[t_{1}, \infty\right)$ is sufficiently large. By Lemma 3 , we have $\ell_{y}^{(0)}>0$, where $\ell_{y}^{(j)}:=\lim _{t \rightarrow \infty} y_{x}^{(j)}(t)$ for $j=0,1, \ldots, n$. Now, we prove that $y_{x}$ is increasing on $\left[t_{2}, \infty\right)$. Suppose the contrary that $y_{x}$ is decreasing on $\left[t_{2}, \infty\right)$, which indicates that $\ell_{y}^{(0)}$ is a positive constant and $\ell_{y}^{(j)}=0$ for $j=1,2, \ldots, n$. As $y_{x} \geq x$ holds on $\left[t_{2}, \infty\right)$, it follows that

$$
x(t) \geq\left(1-p(t) \frac{y_{x}(\tau(t))}{y_{x}(t)}\right) y_{x}(t) \text { for all } t \in\left[t_{2}, \infty\right) .
$$

Using (5) in (4), we have

$$
y_{x}^{(n)}(t)+q(t)\left(1-p(\sigma(t)) \frac{y_{x}(\tau(\sigma(t)))}{y_{x}(\sigma(t))}\right) y_{x}(\sigma(t)) \leq 0 \quad \text { for all } t \in\left[t_{2}, \infty\right) .
$$

Integrating (6) from $t$ to $\infty$ for a total of $(n-1)$ times and integrating the resulting inequality from $t_{2}$ to $\infty$, we have

$$
\int_{t_{2}}^{\infty} \frac{\left(s-t_{2}\right)^{n-1}}{(n-1) !} q(s)\left(1-p(\sigma(s)) \frac{y_{x}(\tau(\sigma(s)))}{y_{x}(\sigma(s))}\right) y_{x}(\sigma(s)) \mathrm{d} s \leq(-1)^{n}\left(\ell_{y}-y_{x}\left(t_{2}\right)\right)<\infty
$$

which implies together with $\ell_{y}^{(0)}>0$ and $(\mathrm{R} 1)$ that

$$
\int_{t_{2}}^{\infty} s^{n-1} q(s)[1-p(\sigma(s))] \mathrm{d} s<\infty
$$

by the result in [13, p. 193], and this indicates

$$
\int_{t_{3}}^{\infty}(\sigma(s))^{n-1} q(s)[1-p(\sigma(s))] \mathrm{d} s<\infty .
$$

However, since every solution of (2) is oscillatory, [14, Theorem 2.6] ensures

$$
\int_{t_{3}}^{\infty}(\sigma(s))^{n-1} q(s)[1-p(\sigma(s))] \mathrm{d} s=\infty,
$$

which contradicts (7); thus we conclude that $y_{x}$ is increasing on $\left[t_{2}, \infty\right)$. From the increasing nature of $y_{x},(5)$ and Lemma 2, we get

$$
x(t) \geq[1-p(t)] y(t) \geq \frac{\lambda_{0}}{(n-1) !} t^{n-1}[1-p(t)] y_{x}^{(n-1)}(t) \text { for all } t \in\left[t_{2}, \infty\right) .
$$

Plugging (8) into (1), we get

$$
y_{x}^{(n)}(t)+\frac{\lambda_{0}}{(n-1) !}(\sigma(t))^{n-1}[1-p(\sigma(t))] q(t) y_{x}^{(n-1)}(\sigma(t)) \leq 0 \quad \text { for all } t \in\left[t_{2}, \infty\right) .
$$

Since $y_{x}^{(n-1)}$ is eventually positive by the Kneser's theorem, we learn from [9, Corollary 3.2.2] that the corresponding differential equation (2) of inequality (9) also has an eventually positive solution. This contradicts the fact that every solution of (2) is oscillatory. The proof is therefore completed. 
REMARK 1 (see [28, Theorem 1]). In addition to the assumptions of Theorem 1 suppose that $n$ is even; then (1) is oscillatory.

Now, we can give the following corollaries.

Corollary 1 (see [9, Theorem 3.1.1] and [11]). Assume that p satisfies the condition (RI). If

$$
\limsup _{t \rightarrow \infty} \int_{\sigma(t)}^{t} \frac{1}{(n-1) !}(\sigma(s))^{n-1}[1-p(\sigma(s))] q(s) \mathrm{d} s>1
$$

or

$$
\liminf _{t \rightarrow \infty} \int_{\sigma(t)}^{t} \frac{1}{(n-1) !}(\sigma(s))^{n-1}[1-p(\sigma(s))] q(s) \mathrm{d} s>\frac{1}{\mathrm{e}}
$$

holds, then (1) is oscillatory when $n$ is even and almost oscillatory when $n$ is odd.

THEOREM 2. Assume that p satisfies the condition (R2), and

$$
y^{\prime}(t)+\frac{\lambda_{0}}{(n-1) !}(\sigma(t))^{n-1} q(t) y(\sigma(t))=0 \quad \text { for } t \in\left[t_{0}, \infty\right)
$$

is oscillatory for some $\lambda_{0} \in(0,1)$. Then (1) is almost oscillatory.

Proof. Let $x$ be a non-oscillatory solution of (1), which does not tend to zero asymptotically. Without loss of generality, we may assume that $x$ is eventually positive. Say $x(t), x(\tau(t)), x(\sigma(t))>0$ for all $t \in\left[t_{1}, \infty\right)$ for some sufficiently large $t_{1} \in\left[t_{0}, \infty\right)$, and set $y_{x}$ for $t \in\left[t_{1}, \infty\right)$ as in (3). Then, we see that (4) holds for all $t \in\left[t_{1}, \infty\right)$, which indicates that $y_{x}^{(j)}$ is strictly monotonic and of single sign on $\left[t_{2}, \infty\right)$ for $j=$ $0,1, \ldots, n-1$, where $t_{2} \in\left[t_{1}, \infty\right)$ is sufficiently large. From Lemma 3 , we learn that $\ell_{y}^{(0)}>0$ holds, where $\ell_{y}^{(0)}:=\lim _{t \rightarrow \infty} y_{x}(t)$, since $x$ does not tend to zero asymptotically. Taking the property $y_{x} \leq x$ into account and proceeding as in the proof of Theorem 1 , we obtain

$$
y_{x}^{(n)}(t)+\frac{\lambda_{0}}{(n-1) !}(\sigma(t))^{n-1} q(t) y_{x}^{(n-1)}(\sigma(t)) \leq 0 \quad \text { for all } t \in\left[t_{2}, \infty\right) .
$$

Since $y_{x}^{(n-1)}$ is eventually positive by Kneser's theorem, we see from [9, Corollary 3.2.2] that the corresponding differential equation (10) of inequality (11) has an eventually positive solution too. This contraction completes the proof.

REMARK 2. In addition to the assumptions of Theorem 2 suppose that $n$ is even, and there exists an increasing divergent sequence $\left\{\xi_{k}\right\}_{k \in \mathbb{N}} \subset\left[t_{0}, \infty\right)$ such that $p\left(\xi_{k}\right)=0$ holds for all $k \in \mathbb{N}$, then (1) is oscillatory.

Corollary 2 (see [9, Theorem 3.1.1] and [11]). Assume that p satisfies the condition (RI). If

$$
\limsup _{t \rightarrow \infty} \int_{\sigma(t)}^{t} \frac{1}{(n-1) !}(\sigma(s))^{n-1} q(s) \mathrm{d} s>1
$$


or

$$
\liminf _{t \rightarrow \infty} \int_{\sigma(t)}^{t} \frac{1}{(n-1) !}(\sigma(s))^{n-1} q(s) \mathrm{d} s>\frac{1}{\mathrm{e}}
$$

holds, then (1) is almost oscillatory.

REMARK 3. Under the assumptions of Theorem 1 and/or Theorem 2, we infer that every unbounded solution of (1) is oscillatory.

REMARK 4. In Corollary 1 and/or Corollary 2, the iterative criteria in [21] can also be applied to improve their results.

4. Applications. Now, we give the following simple examples to illustrate the significance of our results.

EXAMPLE 1 . Let $n \geq 2, p \in[0,1), q \in(0, \infty)$ and $\tau, \sigma \in[1, \infty)$, and consider the following higher-order neutral delay differential equation:

$$
[x(t)+p x(t / \tau)]^{(n)}+\frac{q}{t^{n}} x(t / \sigma)=0 \quad \text { for } t \in[1, \infty) .
$$

Clearly, Theorem B is applicable and Theorem C fails. Indeed, we have

$$
\int^{\infty} s^{n-1} \frac{q}{s^{n}} \mathrm{~d} s=\int^{\infty} \frac{q}{s} \mathrm{~d} s=\infty \quad \text { and } \quad \int^{\infty} s^{n-2} \frac{q}{s^{n}} \mathrm{~d} s=\int^{\infty} \frac{q}{s^{2}} \mathrm{~d} s<\infty .
$$

Hence, we get the concussion of Theorem B that (12) is almost bounded oscillatory. And one can check that [15, Theorems 3.6 and 3.7], [16, Theorems 2.2 and 2.7], [17, Theorem 2.4] and [18, Theorem 2.3] all fail to deliver with unbounded solutions of (12). From Corollary 2 , we see that (12) is almost oscillatory provided that

$$
\liminf _{t \rightarrow \infty} \int_{t / \sigma}^{t} \frac{1}{(n-1) !}\left(\frac{s}{\sigma}\right)^{n-1}[1-p] \frac{q}{s^{n}} \mathrm{~d} s>\frac{1}{\mathrm{e}}
$$

or simply

$$
\frac{\ln \sigma}{\sigma^{n-1}}>\frac{(n-1) !}{\mathrm{e}[1-p] q}
$$

EXAmple 2. Consider the equation in Example 1 with $p \in(-1,0]$; then we see from Corollary 2 that (12) is almost oscillatory provided that

$$
\frac{\ln \sigma}{\sigma^{n-1}}>\frac{(n-1) !}{\mathrm{e} q}
$$

Hence, we again see that Theorem B applies with bounded solutions, and Theorem $\mathrm{C}$ fails to apply. Again, all the results [15, Theorems 3.6 and 3.7], [16, Theorems 2.2 and 2.7], [17, Theorem 2.4] and [18, Theorem 2.3] fail to apply to (12). When $n$ is odd, all the results in $[\mathbf{2}, \mathbf{4}, \mathbf{5}, \mathbf{8}, \mathbf{2 0}, \mathbf{2 7}]$ do not apply because of the delay's form; however, [6] is the only paper that includes applicable results for this equation in some cases. 
5. Final comments. In this section, we talk about some remarks and extensions for the results given in the previous sections.

Our results in Section 3 can also be applied to half-linear equations of the form

$$
[x(t)+p(t) x(\tau(t))]^{(n)}+q(t)|x(\sigma(t))|^{\alpha-1} x(\sigma(t))=0 \quad \text { for } t \in\left[t_{0}, \infty\right),
$$

where $n \geq 2$ is an integer $p, q, \tau, \sigma$ as stated before and $\alpha>0$ is a real number. For such type of equations, one can easily relate oscillatory and/or almost-oscillatory nature of (13) with the same type of first-order equations. The readers may find oscillation results for sub-linear and/or super-linear in [7, 22-24] to give explicit results on (13).

Finally, we would like to point out that [2, Theorems 3 and 4] are not always true; indeed these results always hold only for strictly homogeneous equations (the authors need to assume that the forcing term is eventually non-negative and tends to zero at infinity when giving the proof for eventually negative solutions), and [14, Theorems 2.1-2.3] do not hold for arbitrary delays; i.e. these results hold only for linear delays of slopes not exceeding 1, similar to those in [14, Theorems 2.4-2.6] because of the application of [14, Lemma 1.5] in the proof of [14, Theorem 2.1] (the author had to apply the chain rule, which yields the mistake). Lemma 3 partially salvages [26, Lemma 1], and thus one can easily correct the results in $[3,25,26]$ obtained for the oscillation of second-order neutral delay differential equations.

\section{REFERENCES}

1. R. P. Agarwal, S. R. Grace and D. O'Regan, Oscillation theory for difference and functional differential equations (Kluwer Academic, Dordrecht, 2000).

2. T. Candan and R. S. Dahiya, Oscillatory and asymptotic behavior of odd order neutral differential equations, Dyn. Contin. Discrete Impuls. Syst. Ser. A Math. Anal. 14(6) (2007), 767-774.

3. M. Chen and $\mathrm{Z}$. Xu, Interval oscillation of second-order Emden-Fowler neutral delay differential equations, Electron. J. Diff. Eq. (58) (2007), 9.

4. P. Das, Oscillation in odd-order neutral delay differential equations, Proc. Indian Acad. Sci. Math. Sci. 105(2) (1995), 219-225.

5. P. Das, B. B. Mishra and C. R. Dash, Oscillation theorems for neutral delay differential equations of odd order, Bull. Inst. Math. Acad. Sin. 1(4) (2007) 557-568.

6. J. Džurina, Oscillation theorems for neutral differential equations of higher order, Czechoslovak Math. J. 54(129) (2004), 185-195.

7. L. H. Erbe, Q. Kong and B. G. Zhang, Oscillation theory for functional-differential equations (Marcel Dekker, New York, 1995).

8. K. Gopalsamy, B. S. Lalli and B. G. Zhang, Oscillation of odd order neutral differential equations, Czechoslovak Math. J. 42(2) (1992), 313-323.

9. I. Györi and G. Ladas, Oscillation theory of delay differential equations: With applications (Oxford University Press, New York, 1991).

10. J. K. Hale, Theory of functional differential equations (Springer, New York, 1977).

11. G. Ladas, V. Laskhmikantham and J. S. Papadakis, Oscillations of higher-order retarded differential equations generated by the retarded argument, in Delay and functional differential equations and their applications (Schmitt K., Editor) (Academic, New York, 1972), 219-231.

12. G. S. Ladde, V. Lakshmikantham and B. G. Zhang, Oscillation theory of differential equations with deviating arguments (Marcel Dekker, New York, 1987). 2001).

13. S. C. Mallik and S. Arora, Mathematical analysis (New Age International, New Delhi,

14. N. Parhi and R. N. Rath, Oscillation criteria for forced first order neutral differential equations with variable coefficients, J. Math. Anal. Appl. 256(2) (2001), 525-541.

15. N. Parhi and R. N. Rath, On oscillation of solutions of forced nonlinear neutral differential equations of higher order, Czechoslovak Math. J. 53(128) (2003), 805-825. 
16. N. Parhi and R. N. Rath, On oscillation of solutions of forced nonlinear neutral differential equations of higher order II, Ann. Polon. Math. 81(2), (2003), 101-110.

17. R. N. Rath, Oscillatory and asymptotic behaviour of solutions of higher order neutral equations, Bull. Inst. Math. Acad. Sinica 30(3) (2002), 219-228.

18. R. N. Rath, L. N. Padhy and N. Misra, Oscillation of solutions of non-linear neutral delay differential equations of higher order for $p(t)= \pm 1$, Arch. Math. (Brno) 40(4) (2004), 359-366.

19. Y. Şahiner and A. Zafer, Bounded oscillation of nonlinear neutral differential equations of neutral type, Czechoslovak Math. J. 51(126) (2001) 185-195.

20. J. H. Shen, New oscillation criteria for odd order neutral equations, J. Math. Anal. Appl. 201(2) (1996), 387-395.

21. J. H. Shen and X. H. Tang, New oscillation criteria for linear delay differential equations, Comput. Math. Appl. 36(6) (1998), 53-61.

22. X. H. Tang, Oscillation for first order superlinear delay differential equations, J. Lond. Math. Soc. 65(2), no. 1 (2002), 115-122.

23. L. W. Wang, Oscillation of first-order nonlinear neutral functional-differential equations, Acta Math. Appl. Sinica 14(3) (1991), 348-359.

24. J. J. Wei, Oscillation of first-order sublinear differential equations with deviating arguments, Dongbei Shida Xuebao 3 (1991), 5-9.

25. Z. Xu and X. Liu, Philos-type oscillation criteria for Emden-Fowler neutral delay differential equations, J. Comput. Appl. Math. 206(2) (2007), 1116-1126.

26. Q. Yang, L. Yang and S. Zhu, Interval criteria for oscillation of second-order nonlinear neutral differential equations, Comput. Math. Appl. 46(5-6) (2003), 903-918.

27. B. G. Zhang and W. T. Li, On the oscillation of odd order neutral differential equations. Fasc. Math. 29 (1999), 167-183.

28. Q. Zhang and J. Yan, Oscillation behavior of even order neutral differential equations with variable coefficients, Appl. Math. Lett. 19(11) (2006), 1202-1206. 